\title{
State-trace analysis of the effects of a visual illusion on saccade amplitudes and perceptual judgments
}

\author{
Jason S. MCCARLEY and Christopher Grant \\ University of Illinois at Urbana-Champaign, Urbana, Illinois
}

\begin{abstract}
Visual illusions often appear to have a larger influence on subjective judgments than on visuomotor behavior. Although this effect has been taken as evidence for multiple estimates of stimulus size in the visual brain, dissociations between subjective judgments and visuomotor measures can frequently be reconciled with a singleestimate model. To circumvent this difficulty, we used state-trace analysis in a pair of experiments to examine the effects of the Müller-Lyer illusion on subjective length estimates, voluntary saccade amplitudes, and reflexive saccade amplitudes. All dependent measures were affected by the illusion. However, state-trace analyses revealed nonmonotonic relationships among all three variables, a pattern inconsistent with the possibility of a single underlying estimate of stimulus size.
\end{abstract}

Object recognition and visuomotor control impose different and largely complementary demands on visual processing (Ganel \& Goodale, 2003; Milner \& Goodale, 2006). Recognition requires the ability to match stimulus information to stored patterns, despite variations in the retinal image across viewpoints. Control of visually guided behavior, conversely, requires sensitivity to metric information across changes in viewing distance and angle. In light of these differences, several researchers have suggested that visual perception and action are rooted in separable neural systems, employing distinct forms of stimulus representation. One encodes object-centered representations, largely insensitive to changes in viewing angle and distance. The other encodes rapidly updated object representations in viewer-centered coordinates. Input from the dual representations converges to shape perception and behavior as task demands necessitate, with object-centered representations dominating conscious perceptual judgments and viewer-centered representations dominating visuomotor control (Bridgeman, 1999; Goodale \& Milner, 1992; see Goodale \& Westwood, 2004, for a review).

Evidence for the two-visual-systems hypothesis has come in part from studies comparing the effects of illusions on perceptual judgments and visuomotor behavior. Consistent with the speculation that perception and action are informed by multiple spatial representations, several authors have reported that illusions evident in subjective reports of stimulus size often have little influence on visually guided actions. Aglioti, DeSouza, and Goodale (1995) and Haffenden and Goodale (1998), for example, found larger effects of the Titchener illusion on perceptual judgments than on grip scaling. Similarly, Bridgeman, Peery, and Anand (1997) found an effect of the Roelofs illusion on psychophysical judgments but not on visually guided pointing, and Wong and Mack (1981) found no influence of a motion illusion on saccades to a flashed target. As predicted by the claim of multiple interacting representations, furthermore, researchers have demonstrated that the influence of an illusion on visually guided behavior varies with task demands. Vishton et al. (2007), for instance, found that preparing to reach for an object reduced the effects of an illusion on perceptual judgments, and in a study of eye movements, McCarley, Kramer, and DiGirolamo (2003; see also DiGirolamo, McCarley, Kramer, \& Griffin, 2008) found that the Müller-Lyer (M-L) illusion had larger effects on perceptual judgments and on voluntary saccadesthat is, eye movements generated in the absence of an exogenous visual cue (Henik, Rafal, \& Rhodes, 1994; Klein, Kingstone, \& Pontefract, 1992) - than on reflexive saccades - that is, movements generated in response to a transient visual signal.

In the terminology of Loftus, Oberg, and Dillon (2004), an account postulating separate representations for control of perception and action constitutes a twodimensional model. The behaviors of interest, that is, are mediated by two latent values. In theories positing dissociable effects of an illusion on perception and visually guided behaviors, one of these is presumed to be an estimate of stimulus size biased by the illusion, and the second is presumed to be an unbiased estimate. Subjective judgments and visuomotor behaviors are hypothesized to be differentially influenced by these values, then, and

J. S. McCarley, mccarley@illinois.edu 
evidence of the dual inputs is inferred from statistical interactions between the presence and/or direction of the illusion and the modality of the response (subjective or perceptual-motor). Unfortunately, such an interaction can often be reconciled with a unidimensional account as well. If perceptual and motor tasks are not appropriately matched, for example, an illusion may affect one form of response more than the other even if both responses are mediated by a common representation (e.g., Franz, Gegenfurtner, Bülthoff, \& Fahle, 2000; see Amazeen \& DaSilva, 2005, and Haffenden \& Goodale, 2000, for a discussion of the converse problem, that of coincidental similarity between perceptual and motor responses). Differences between the stimuli for perceptual and motor tasks, or for different forms of motor task, may also produce specious dissociations. In comparing the influence of the M-L illusion on reflexive and voluntary saccades, for instance, the exogenous signal used to elicit reflexive movements might diminish the influence of the illusion even within a unidimensional model. In such a case, a single estimate of stimulus length, susceptible to the illusion, would determine the value of all saccade amplitudes, but the effects of the illusion would be greater for voluntary than for reflexive movements because the exogenous signal used to trigger a reflexive movement would attenuate the influence of the stimulus wings on the estimated length. Moreover, if psychophysical judgments were made in the absence of an exogenous signal or were based on information processed before or after the exogenous signal, they would show an effect of the illusion similar in magnitude to that of the voluntary movements and greater than that of the reflexive movements.

Thus, differences in the effect of an illusion on actions and perceptual judgments, or on different forms of action, do not necessarily disconfirm a unidimensional perception-action model. Uni- and multidimensional models can be distinguished, however, through statetrace analysis (Bamber, 1979; Dunn \& Kirsner, 1988; Harley, Dillon, \& Loftus, 2004; Loftus et al., 2004). In a state-trace analysis, one dependent variable is plotted as a function of another. In a study of learning, for example, item recall might be plotted as a function of item recognition (Dunn \& Kirsner, 1988); in the present study, saccade amplitudes are plotted as a function of subjective size estimates. Of interest is the form of the relationship between the dependent variables. In a unidimensional model, both vary as functions of a single latent variable. Under the weak assumption that the relationship between the latent and observable variables is monotonic, any manipulation that increases the value of the latent variable will also increase the value of both dependent variables. Consequently, the relationship between the dependent measures will itself be monotonic. A nonmonotonic relationship (sometimes called a reversed association; Dunn \& Kirsner, 1988) thus disconfirms a unidimensional model, demonstrating that at least two underlying variables are necessary to account for the relationship between dependent measures ${ }^{1}$ (Loftus et al., 2004; see the Appendix of Harley et al., 2004, for a lucid and brief introduction to state-trace analysis). Moreover, this conclusion is invariant over any monotonic transformation of the dependent variables (Harley et al., 2004). In the present context, state-trace analysis thus offers the benefit that it does not require careful matching of perceptual and motor-response tasks.

Notably, a reanalysis of earlier data (McCarley et al., 2003) comparing the effects of the M-L illusion on voluntary and reflexive saccades produced evidence of a monotonic relationship consistent with a unidimensional model of saccade amplitude programming. The goal of the present study was to discriminate between these two classes of model using state-trace analysis. Stimuli were wings-in $(\longleftrightarrow)$ and wings-out $(><$ M-L figures varying in length. Observers in each experiment performed three tasks. In the first task, the observer made a voluntary saccade from one end of the stimulus figure to the other. In the second, the observer made a reflexive saccade from one end of the figure to the other. Finally, in the third, the observer provided a subjective judgment of stimulus length. The relationship between dependent variables was examined through state-trace analyses as described above. In Experiment 1, subjective judgments were reported using a category rating procedure (Gescheider, 1997), in which observers assigned a number (1-9) to describe the perceived horizontal length of each stimulus. In Experiment 2, subjective reports were made using a magnitude estimation task (Gescheider, 1997; Stevens, 1975). Both of these techniques, notably, produce subjective reports on a different scale than eye movement amplitudes. Nonetheless, reported values in both cases are expected to increase monotonically with perceived stimulus length and, thus, to meet the assumption necessary for state-trace analysis.

\section{EXPERIMENT 1}

In Experiment 1, we examined the relationships among voluntary saccade amplitudes, reflexive saccade amplitudes, and perceptual judgments of stimulus size, where perceptual judgments were rendered through a simple 1-9 category rating.

\section{Method}

Observers. Observers were 12 young adults with normal or corrected-to-normal visual acuity.

Apparatus. Stimuli were presented on a 22 -in. monitor with resolution of $1,024 \times 768$ pixels and a refresh rate of $60 \mathrm{~Hz}$. Eye movements were recorded with an EyeLink II eyetracking system (SRI Ltd., Mississaugua, ON) with a sampling rate of $250 \mathrm{~Hz}$. Displays were viewed from a distance of $75 \mathrm{~cm}$, controlled by a cheekrest.

Stimuli. The stimuli were horizontal M-L figures. The wings of the figure were $1.5^{\circ}$ in length and oriented $\pm 30^{\circ}$, relative to the horizontal shaft. The length of the shaft varied from $5.8^{\circ}$ to $8.6^{\circ}$ in steps of approximately $0.57^{\circ}$. The stimuli were drawn in gray against a black background.

Procedure. Each observer performed three tasks. In the reflexive and voluntary saccade tasks, the observer made a saccade on each trial from the left to the right endpoint of the stimulus shaft. The observer began a trial by gazing at a central fixation mark and 
pressing a button on a handheld controller. The fixation mark was then removed, and the stimulus figure appeared, with the vertex of its left wing at the fixation mark. The saccade go signal was presented $500 \mathrm{msec}$ later. In the voluntary saccade task, the signal was a $200-\mathrm{msec}, 500-\mathrm{Hz}$ tone that originated from speakers on either side of the visual display. In the reflexive saccade task, the signal was a white $0.2^{\circ} \times 0.2^{\circ}$ rectangle that appeared for $50 \mathrm{msec}$ at the saccade target location. In both tasks, observers were asked to make saccades as quickly as possible following the go signal. The M-L stimulus remained visible for $500 \mathrm{msec}$ following saccade initiation, at which time the trial ended.

In the subjective judgment task, the observer again began a trial by gazing at the fixation mark and pressing a button, after which the stimulus for the trial appeared for an exposure duration of $500 \mathrm{msec}$ and was removed. The observer's task was to provide a 1-9 rating of the perceived stimulus length by pressing a key on the computer's numeric keypad.

Observers performed 11 blocks of trials per task. Each block comprised 12 trials, 1 for each combination of stimulus configuration and length. The order of trials was randomized within blocks. The order of tasks was counterbalanced across observers.

\section{Results}

Data were discarded from trials on which an eye movement occurred within $500 \mathrm{msec}$ following M-L figure onset, both in the saccade tasks and in the subjective-judgment task. This ensured that in all three tasks, the observer's gaze remained fixed on the left endpoint of the stimulus figure until the time of the response signal. Effects of the M-L illusion on eye movements were examined by measuring the horizontal amplitude of the first saccade that occurred following a go signal. To exclude anticipatory and inattentive saccades, eye movement data were discarded from trials on which the first saccade occurred with a latency of less than $50 \mathrm{msec}$ or greater than $500 \mathrm{msec}$ and on trials for which the first saccade had a horizontal amplitude of less than 50 pixels in the correct direction. Mean saccade latency was $271 \mathrm{msec}(S E=11.35)$ for voluntary movements and $226 \mathrm{msec}(S E=11.83)$ for reflexive movements $[t(11)=$ $5.087, p<.001]$.

Figure 1 presents mean category ratings (left), along with mean amplitudes for voluntary (middle) and reflexive (right) saccades. For preliminary analysis, category rating data were submitted to a $2 \times 6$ ANOVA with stimulus length and stimulus configuration (wings in vs. wings out) as within-subjects factors. Reported $p$ values and degrees of freedom $(d f \mathbf{s})$ for all analyses reflect Greenhouse-Geisser correction where appropriate. Reliable main effects indicated that category ratings increased with stimulus length $\left[F(2.315,25.460)=153.158, M S_{\mathrm{e}}=\right.$ $\left..444, p<.001, \eta_{\mathrm{p}}^{2}=.933\right]$ and were higher for wingsout than for wings-in stimuli $\left[F(1,11)=99.296, M S_{\mathrm{e}}=\right.$ $\left.1.157, p<.001, \eta_{\mathrm{p}}^{2}=.900\right]$. The data thus confirmed that the stimulus manipulations affected category ratings in the expected manner.

Preliminary analysis of eye movement data was performed with a $2 \times 2 \times 6$ ANOVA with saccade type (voluntary vs. reflexive), stimulus length, and stimulus configuration as within-subjects factors. Analysis confirmed that saccade amplitudes increased with stimulus length $\left[F(3.612,39.728)=595.868, M S_{\mathrm{e}}=.128, p<.001, \eta_{\mathrm{p}}^{2}=\right.$ $.982]$ and were longer for wings-out than for wings-in stimuli $\left[F(1,11)=134.321, M S_{\mathrm{e}}=.307, p<.001, \eta_{\mathrm{p}}^{2}=\right.$ $.924]$. Additionally, analyses conducted separately for the voluntary and reflexive saccade data confirmed that the influence of the illusion on saccade amplitude was reliable in both cases $(p \mathrm{~s}<.001)$. The omnibus analysis also revealed a highly reliable interaction of saccade type $\times$ stimulus configuration $\left[F(1,11)=55.727, M S_{\mathrm{e}}=.109\right.$, $\left.p<.001, \eta_{\mathrm{p}}^{2}=.835\right]$, however, replicating earlier findings that the effect of the illusion was smaller for reflexive than for voluntary movements.

The results thus far demonstrate effects of the M-L illusion on all three measures. State-trace analyses were conducted to determine whether the relationships between measures were consistent with a single underlying estimate of stimulus size or gave evidence of multiple estimates. Figure 2 presents plots of voluntary saccade amplitudes versus subjective judgments (left), reflexive saccade amplitudes versus subjective judgments (middle), and reflexive versus voluntary saccade amplitudes (right). The data are plotted separately for the wings-in and wings-out stimuli within each graph. As is clear from inspection, the relationship
Subjective Reports

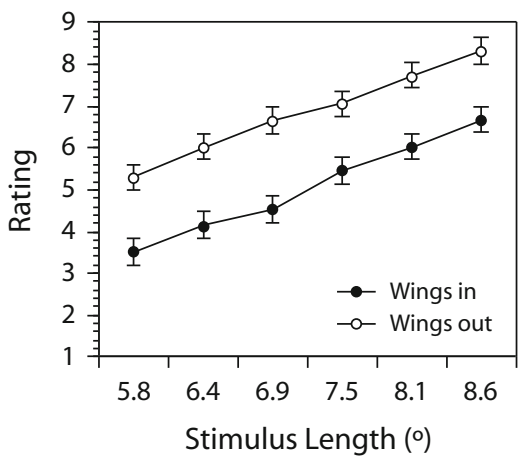

Voluntary Saccades

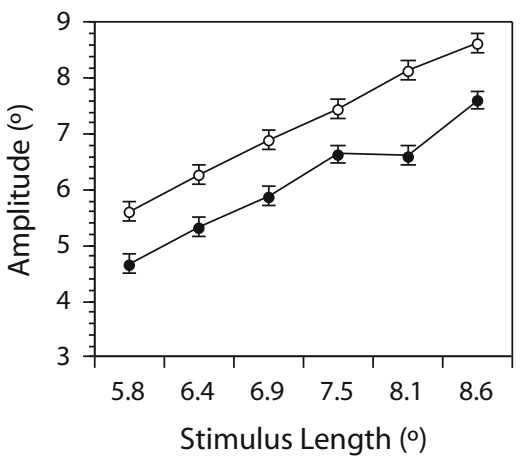

Reflexive Saccades

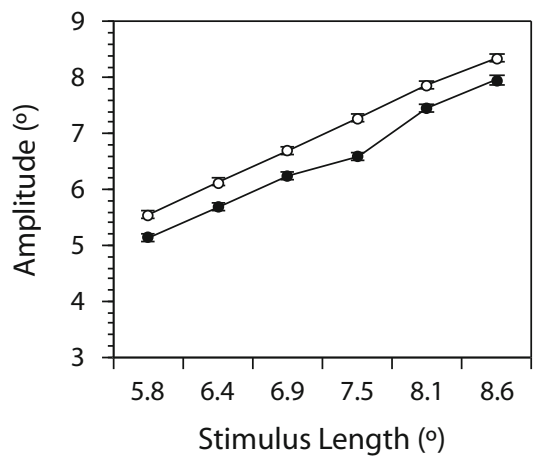

Figure 1. Mean subjective ratings of stimulus length (left), voluntary saccade amplitudes (middle), and reflexive saccade amplitudes (right) of Experiment 1. Error bars in all figures indicate within-subjects standard errors based on the main effect of stimulus configuration (wings in vs. wings out; Loftus \& Masson, 1994). 

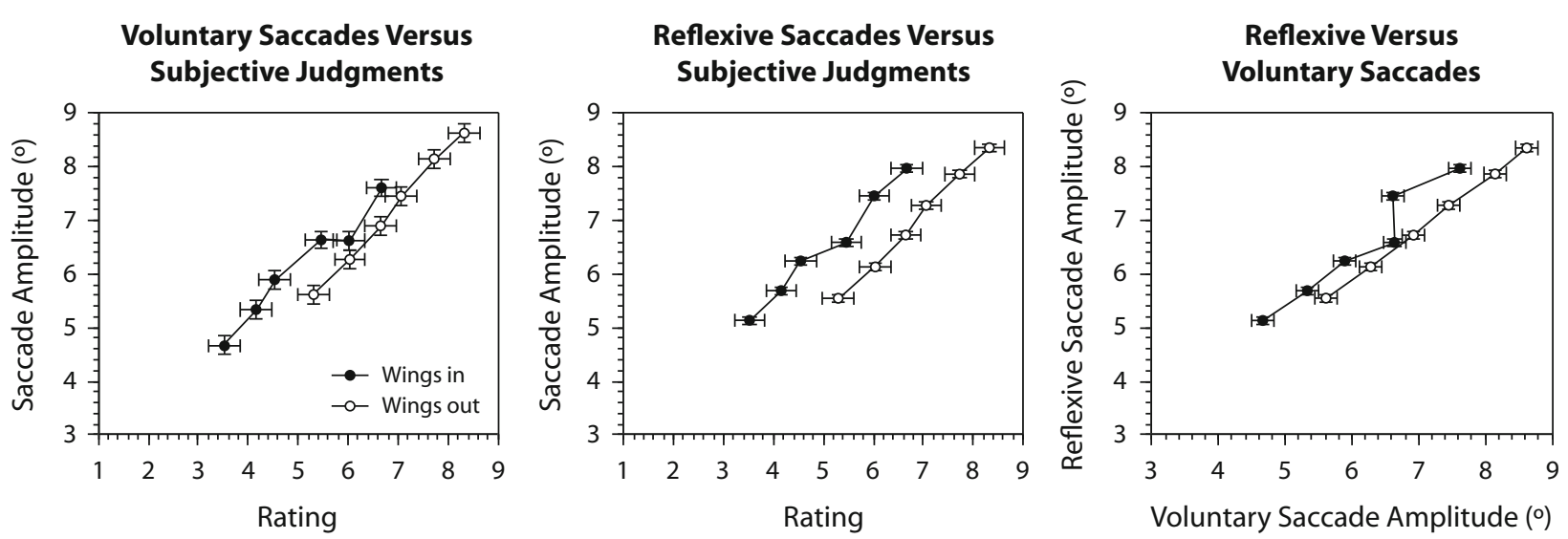

Figure 2. State-trace plots of the data of Experiment 1: voluntary saccade amplitudes versus subjective reports (left), reflexive saccade amplitudes versus subjective reports (middle), and reflexive saccade amplitudes versus voluntary saccade amplitudes (right).

between variables is nonmonotonic in all three plots; in all cases, wings-in and wings-out data form distinct curves, rather than a single monotonically increasing function. The data thus suggest that no two pairs of independent variables were informed by a single, common estimate. In Experiment 2, we sought further evidence for this conclusion.

\section{EXPERIMENT 2}

The results of the first experiment appeared to disconfirm unidimensional models of the relationship between voluntary and reflexive saccade programming and between either form of saccade programming and perceptual judgments. In Experiment 2, we sought additional evidence for these conclusions using an alternative form of perceptual judgment task. Here, observers provided length judgments through a magnitude estimation procedure (Gescheider, 1997; Stevens, 1975), giving numeric estimates of stimulus length relative to an arbitrary standard.

\section{Method}

Observers. The observers were 12 young adults with normal or corrected-to-normal visual acuity.
Apparatus and Stimuli. The apparatus and stimuli were identical to those of Experiment 1.

Procedure. The procedure was identical to that of Experiment 1, except for a change in the method by which observers provided perceptual judgments. Before beginning the perceptual judgment task, observers were shown a horizontal line segment of $7.2^{\circ}$, equal in length to the average of the shortest and longest segments used as stimuli. Observers were asked to assign this segment a length of 100 , and then to report the length of the stimulus presented on each trial relative to this standard value.

\section{Results}

The treatment of data and statistical analysis were identical to those of Experiment 1. Unexpectedly, the mean latency was numerically shorter for voluntary saccades $(M=232 \mathrm{msec}, S E=14)$ than for reflexive movements $(M=254 \mathrm{msec}, S E=15)[t(11)=1.722, p=.112]$. This result contrasts with the common finding that reflexive saccades are initiated faster than voluntary saccades, as we found in Experiment 1. The reason for this anomaly is unclear. Additional results, however, were consistent with those reported for Experiment 1.

The data are presented in Figure 3. Analysis of the perceptual judgments indicated that magnitude estimates in-
Subjective Reports

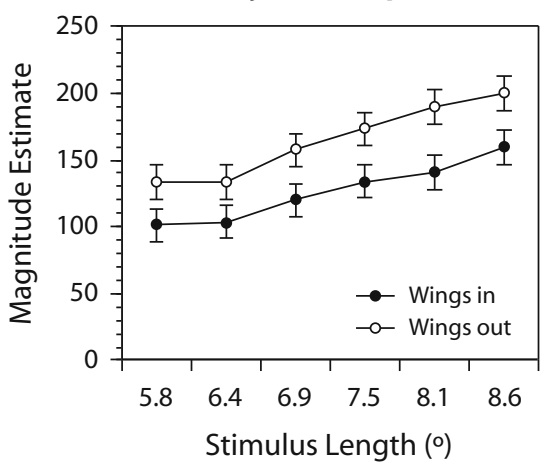

Voluntary Saccades

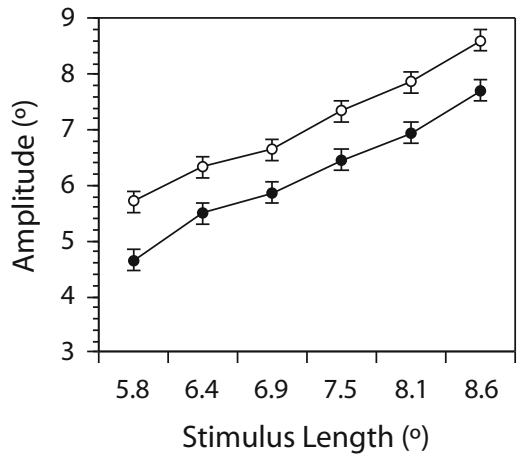

Reflexive Saccades

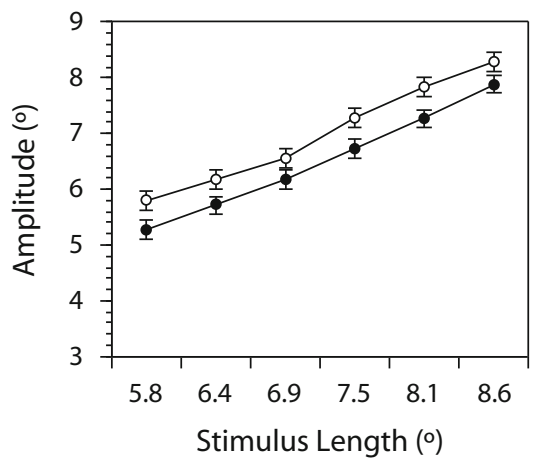

Figure 3. Mean magnitude estimates (left), voluntary saccade amplitudes (middle), and reflexive saccade amplitudes (right) of Experiment 2. 

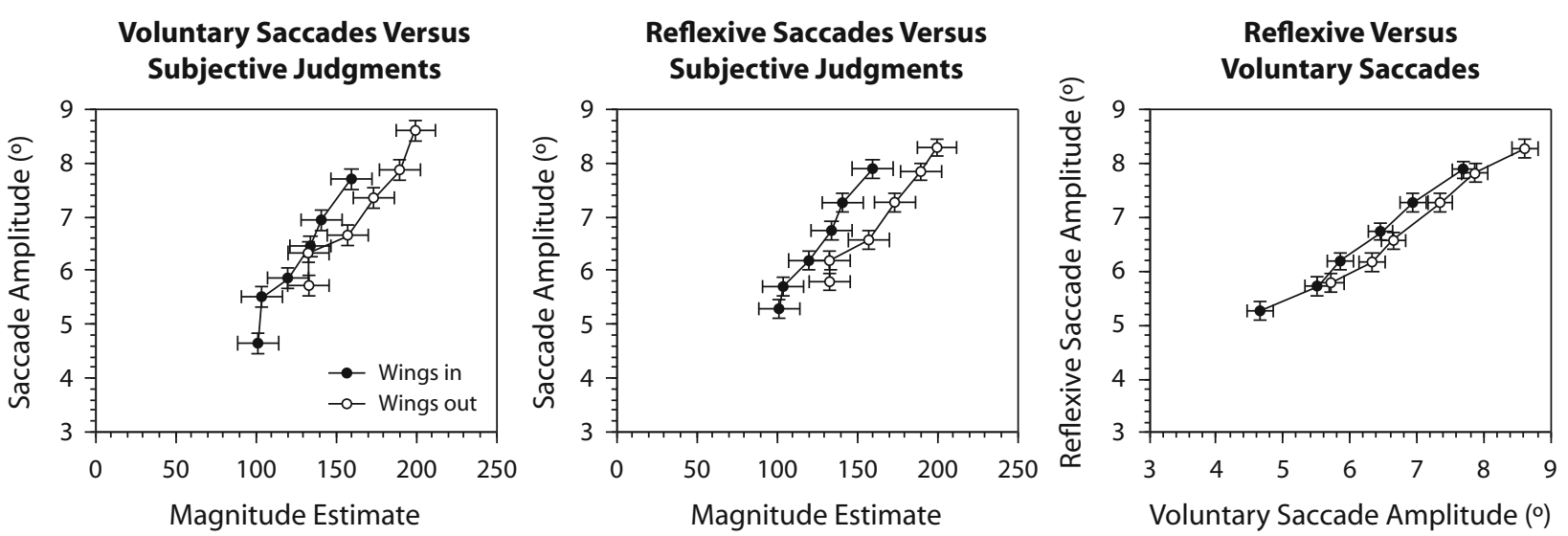

Figure 4. State-trace plots of the data of Experiment 2.

creased with stimulus length $[F(1.758,19.341)=28.561$, $\left.M S_{\mathrm{e}}=1,523.708, p<.001, \eta_{\mathrm{p}}^{2}=.722\right]$ and were higher for wings-out than for wings-in stimuli $[F(1,11)=27.247$, $\left.M S_{\mathrm{e}}=1,902.308, p<.001, \eta_{\mathrm{p}}^{2}=.712\right]$. Analysis of the eye-movement data revealed likewise that saccade amplitudes increased with stimulus length $[F(2.856,31.417)=$ $\left.515.962, M S_{\mathrm{e}}=.171, p<.001, \eta_{\mathrm{p}}^{2}=.979\right]$ and were longer for wings-out than for wings-in stimuli $[F(1,11)=$ $\left.54.900, M S_{\mathrm{e}}=.617, p<.001, \eta_{\mathrm{p}}^{2}=.833\right]$, and analyses conducted separately for the voluntary and reflexive saccade data confirmed that the influence of wing orientation on amplitude was reliable for both forms of saccade ( $p$ s $<$ $.001)$. Omnibus analysis also produced a reliable interaction of saccade type $\times$ stimulus configuration $[F(1,11)=$ $\left.19.352, M S_{\mathrm{e}}=.161, p=.001, \eta_{\mathrm{p}}^{2}=.638\right]$, however, again confirming that the illusion had stronger effects on voluntary than on reflexive saccades.

Figure 4 presents state-trace plots of the data of Experiment 2. Consistent with the results of Experiment 1, the data for wings-in and wings-out conditions form distinct functions in plots of saccade amplitude versus subjective length estimates, evincing clear nonmonotonic relationships between the plotted variables. Curves for the wingsin and wings-out functions in the plot of reflexive versus voluntary saccade amplitudes are less clearly separated than those in the data of Experiment 1. The wings-in curve, though, is again shifted consistently to the left of the wings-out curve. The results therefore appear once more to be incompatible with a unidimensional model of perception and saccade programming.

\section{CROSS-EXPERIMENT ANALYSES}

State-trace analyses in both Experiments 1 and 2 found nonmonotonic relationships among voluntary saccade amplitudes, reflexive saccade amplitudes, and perceptual judgments of stimulus length. As a check on the validity of the state-trace methodology applied here, we conducted an additional analysis to examine the relationship between subjective measures across experiments. By the reasoning of state-trace analysis, two measures that reflect a single, common latent variable should be monotonically related. This implies that the length ratings of Experiment 1 and the magnitude estimates of Experiment 2, if they are measures of a common value of perceived stimulus length, should form a monotonic function when plotted against one another. Inspection of Figure 5 reveals that the data indeed conform to this prediction.

To increase statistical power, finally, we combined the saccade data from Experiments 1 and 2 for analysis. Figure 6 presents a state-trace of reflexive versus voluntary movement amplitudes, incorporating data from both experiments. The wings-in and wings-out data again show a clear nonmonotonic relationship between measures.

\section{GENERAL DISCUSSION}

The present results replicate earlier findings that voluntary and reflexive saccades are differently susceptible

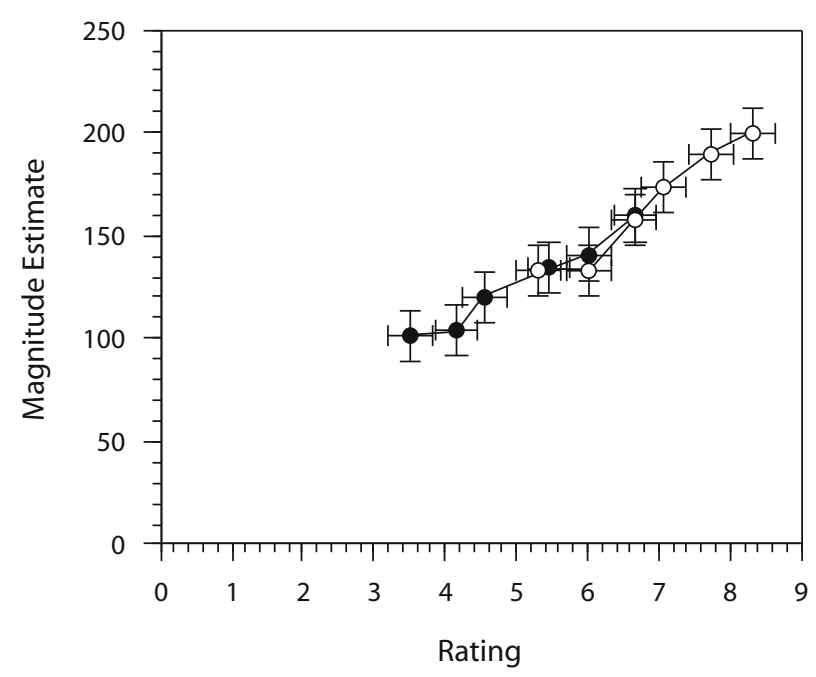

Figure 5. State-trace plot relating the magnitude estimates of Experiment 2 to the length ratings of Experiment 1. 


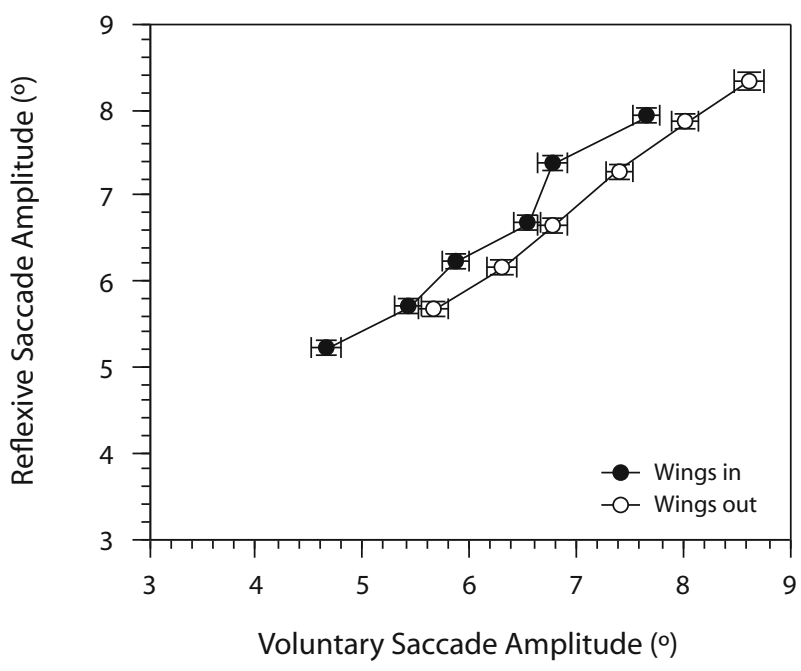

Figure 6. State-trace plot of the combined saccade data of Experiments 1 and 2.

to the M-L illusion and, more importantly, demonstrate that these effects cannot be accommodated by a unidimensional model of voluntary and reflexive movement programming. Across two experiments, state-trace analyses revealed nonmonotonic relationships between voluntary and reflexive saccade amplitudes, a pattern of effects incompatible with a model in which the two forms of movement are programmed on the basis of a single, common estimate of saccade target distance. The data also indicate that multiple estimates are necessary to explain the relationship between perceptual estimates of stimulus size and eye-movement amplitudes: Analyses revealed nonmonotonic relationships between perceptual judgments and saccade amplitudes, both when saccades were voluntary and when they were reflexive.

Altogether, these results are consistent with the two-visual-systems hypothesis, which posits separate but interacting representations for the control of conscious perception and visually guided behavior (Milner $\&$ Goodale, 2006). One potential interpretation of the present data is that a representation immune to the M-L illusion participates in saccade programming but does not influence subjective estimates of stimulus size. A second representation, susceptible to the illusion, underlies the subjective perception of illusive size differences produced by inward- and outward-pointing wings and allows the errors of saccade programming produced by illusive size changes. The input of the second representation to the programming of saccade amplitudes, though, is weighted differently for voluntary and reflexive movements, with input from the illusion-susceptible representation being weighted heavily in voluntary saccade programming but only modestly in reflexive saccade programming. This proposal contradicts earlier speculation that subjective perception and voluntary saccade programming are informed by a single representation (McCarley et al., 2003) but accords with the suggestion that visually guided behavior results from the interplay of representations. Further research will obviously be necessary to test the present account further. Additional work will also be needed, of course, to identify the neural loci of the representations under study. One plausible possibility is that the effects of the illusion arise in the visual cortex (Weidner \& Fink, 2007) and influence saccade programming via backprojections from the cortex to the superior colliculus, whereas the moderating influence of retinal size on eye movements is produced by direct connections from the retina to the colliculus (Pierrot-Deseilligny, Milea, \& Müri, 2004).

\section{AUTHOR NOTE}

Thanks to Andrew Heathcote and Geoffrey Loftus for helpful comments on an earlier draft of this article. Correspondence concerning this article should be addressed to J. S. McCarley, University of Illinois, Institute of Aviation, \#1 Airport Rd., Savoy, IL 61874 (e-mail: mccarley@, illinois.edu).

\section{REFERENCES}

Aglioti, S., DeSouza, J. F. X., \& Goodale, M. A. (1995). Sizecontrast illusions deceive the eye but not the hand. Current Biology, 5, 679-685

Amazeen, E. L., \& DaSilva, F. (2005). Psychophysical test for the independence of perception and action. Journal of Experimental Psychology: Human Perception \& Performance, 31, 170-182.

BAMBER, D. (1979). State-trace analysis: A method of testing simple theories of causation. Journal of Mathematical Psychology, 19, 137-181.

BRIDGEMAN, B. (1999). Separate representations of visual space for perception and visually guided behavior. In G. Aschersleben, T. Bachmann, \& J. Müsseler (Eds.), Cognitive contributions to the perception of spatial and temporal events (pp. 3-13). Amsterdam: Elsevier.

Bridgeman, B., PeERy, S., \& AnAND, S. (1997). Interaction of cognitive and sensorimotor maps of visual space. Perception \& Psychophysics, 59, 456-469.

DiGirolamo, G. J., McCarley, J. S., Kramer, A. F., \& Griffin, H. J. (2008). Voluntary and reflexive eye movements to illusory lengths. Visual Cognition, 16, 68-89.

DunN, J. C., \& Kirsner, K. (1988). Discovering functionally independent mental processes: The principle of reversed association. Psychological Review, 95, 91-101.

Franz, V. H., Gegenfurtner, K. R., Bülthoff, H. H., \& Fahle, M. (2000). Grasping visual illusions: No evidence for a dissociation between perception and action. Psychological Science, 11, 20-25.

Ganel, T., \& Goodale, M. A. (2003). Visual control of action but not perception requires analytical processing of object shape. Nature, 426, 664-667.

Gescheider, G. A. (1997). Psychophysics: The fundamentals. Mahwah, NJ: Erlbaum.

Goodale, M. A., \& Milner, A. D. (1992). Separate visual pathways for perception and action. Trends in Neurosciences, 15, 20-25.

Goodale, M. A., \& Westwood, D. A. (2004). An evolving view of duplex vision: Separate but interacting cortical pathways for perception and action. Current Opinion in Neurobiology, 14, 203-211.

Haffenden, A. M., \& Goodale, M. A. (1998). The effect of pictorial illusion on prehension and perception. Journal of Cognitive Neuroscience, 10, 122-136.

Haffenden, A. M., \& Goodale, M. A. (2000). Independent effects of pictorial displays on perception and action. Vision Research, $\mathbf{4 0}$, 1597-1607.

Harley, E. M., Dillon, A. M., \& Loftus, G. R. (2004). Why is it difficult to see in the fog? How stimulus contrast affects visual perception and visual memory. Psychonomic Bulletin \& Review, 11, 197-231.

Henik, A., RAFAl, R., \& Rhodes, D. (1994). Endogenously generated and visually guided saccades after lesions of the human frontal eye fields. Journal of Cognitive Neuroscience, 6, 400-411. 
Klein, R. M., Kingstone, A., \& Pontefract, A. (1992). Orienting of visual attention. In K. Rayner (Ed.), Eye movements and visual cognition: Scene perception and reading (pp. 46-65). New York: Springer. LofTus, G. R., \& Masson, M. E. J. (1994). Using confidence intervals in within-subject designs. Psychonomic Bulletin \& Review, 1, 476-490.

Loftus, G. R., OberG, M. A., \& Dillon, A. M. (2004). Linear theory, dimensional theory, and the face-inversion effect. Psychological Review, 111, 835-863.

McCarley, J. S., Kramer, A. F., \& DiGirolamo, G. J. (2003). Differential effects of the Müller-Lyer illusion on reflexive and voluntary saccades. Journal of Vision, 3, 751-760.

Milner, A. D., \& Goodale, M. A. (2006). The visual brain in action (2nd ed.). Oxford: Oxford University Press.

Pierrot-Deseilligny, C., Milea, D., \& Müri, R. M. (2004). Eye movement control by the cerebral cortex. Current Opinion in Neurology, 17, 17-25.

Stevens, S. S. (1975). Psychophysics: Introduction to its perceptual, neural, and social prospects. New Brunswick, NJ: Wiley.
Vishton, P. M., Stephens, N. J., Nelson, L. A., Morra, S. E., Brunick, K. L., \& SteVEns, J. A. (2007). Planning to reach for an object changes how the reacher perceives it. Psychological Science, 18, 713-719.

WEIDNER, R., \& FINK, G. R. (2007). The neural mechanisms underlying the Müller-Lyer illusion and its interaction with visuospatial judgments. Cerebral Cortex, 17, 878-884.

Wong, E., \& MAck, A. (1981). Saccadic programming and perceived location. Acta Psychologica, 48, 121-131.

\section{NOTE}

1. By happenstance, a pair of dependent variables may be monotonically related even within a multidimensional model. A nonmonotonic relationship, however, cannot be reconciled with a unidimensional model.

(Manuscript received November 13, 2007; revision accepted for publication April 13, 2008.) 